\title{
Managed-Care-Vorlage: Basisdemokratie und Hausärzte Schweiz
}

\section{Ärztegesellschaft \\ Baselland}

La version française suivra dans le prochain numéro.
Geschätzte Kolleginnen und Kollegen

Am 3. November haben sich die 42 Delegierten von Hausärzte Schweiz (MFE) mit 3/4-Mehrheit dafür entschieden, das von der FMH und verschiedenen Ärzteorganisationen getragene Referendum gegen die Managed-Care-Vorlage nicht zu unterstützen und in einem Pro-Komitee mitzumachen. Dieser Entscheid provoziert uns zum folgenden Kommentar:

Die Ärztegesellschaft Baselland hatte zusammen mit den drei weiteren Kantonalgesellschaften als Initianten der Urabstimmung und der fmCh Mitte September, somit vor Ende der Urabstimmung und vor Bekanntgabe des Resultates, einen vorbehaltenen Entschluss gefasst. Inhalt: Im Falle eines Nein (auch wenn denkbar knapp mit 1\% oder einer Stimme) werden wir das Resultat akzeptieren, der FMH gratulieren, deren Kurs in dieser Sache mittragen und insbesondere keinem Referendumskomitee beitreten. Begründung: 1. Die Einheit der Ärzteschaft und das nach aussen geschlossene Auftreten der FMH ist weitaus höher zu gewichten als die Interessen einer Organisation oder einer Gruppe von Gesellschaften in einer einzelnen Sachfrage. 2. Eine Urabstimmung $\mathrm{zu}$ verlangen und sich dann um das Resultat zu foutieren, ist ein «No go».

Es hat sich gezeigt, dass bis auf die Initianten alle Führungsstrukturen der FMH (Präsident, Zentralvorstand, Ärztekammer, die meisten Vorstände der Kantonalgesellschaften/KKA) die Befindlichkeit der Basis in dieser Sache falsch eingeschätzt hatten. Es spricht ärzte beider Basel) eine 3/4-Zustimmung zum Referendum ergeben, somit eine deutlichere Ablehnung der Vorlage als bei der Urabstimmung im «Gemischtwarenladen» FMH. Gleiches Resultat bei den Médecins de Famille de Genève. Uns ist keine Organisation bekannt, bei der eine Mitgliederumfrage eine Zustimmung zur Vorlage ergeben hätte. Stimmt die minutiöse Analyse unserer (Hausarzt-)Spezialisten in BaselStadt und Baselland, birgt die Vorlage inhaltliche Gefahren, indem zum Beispiel keineswegs festgeschrieben ist, dass die Steuerung durch die Hausärzte zu erfolgen hat, und die erhoffte Aufwertung der Hausarztmedizin somit fraglich ist. Das Parlament hat mit dieser Vorlage das Ziel, letztendlich ein Globalbudget einzurichten.

Allzu oft haben wir Gräben zwischen Hausärzten und Spezialisten, und es ist auf kantonaler Ebene eine unserer zentralen Führungsaufgaben, diese Gräben zuzuschütten, Brücken zu bauen und das Verständnis zwischen den verschiedenen Interessengruppierungen zu verbessern. Für ein Mal gibt es nun keine Gräben zwischen Hausärzten und Spezialisten, und das Misstrauen gegen diese Managed-Care-Vorlage und das Parlament sowie der Widerstand gegen diese Vorlage ziehen sich quer durch alle Fachgruppen, Generationen und Kantone.

Die hilflosen Versuche der Politiker, die Gegner der Vorlage zu diffamieren, als ewiggestrige Allesverhinderer, als innovationsfeindlich darzustellen, von «unheiligen Allianzen» zu sprechen und eine Spaltung der Ärzteschaft herbeizureden, zeigt auf, wie

\section{«Für ein Mal gibt es nun keine Gräben zwischen Hausärzten und Spezialisten.»}

Korrespondenz: Dr. med. Tobias Eichenberger Präsident Ärztegesellschaft Baselland

FMH Urologie/

Operative Urologie

Hammerstrasse 35

CH-4410 Liestal aber auch für eine hohe Professionalität und ein intaktes Demokratieverständnis der genannten Personen und Organisationen, dass der Entscheid der Basis vorbehaltlos akzeptiert wurde und dass die Ärztekammerdelegierten am 26. Oktober (zum Teil sicher «contre cœur») mit jeweils grossem Mehr die Grundsteine für eine erfolgreiche Referendumskampagne gelegt haben.

Bei uns hat eine kürzlich durchgeführte Befragung der Mitglieder des VHBB (Vereinigung Haus- sehr sich das Parlament fürchtet, einer zwar vielseitig interessierten, aber nach aussen geschlossen auftretenden und patientennah und somit auch stimmbürgernah politisierenden Ärzteschaft gegenüberzustehen, die ihre Referendumsfähigkeit beweist und dabei ist, nach dem Primat der Medizin auch das Primat der Gesundheitspolitik anzustreben. Wir alle wissen, wie flexibel und innovativ sich unsere Ärzte den stetig wechselnden Herausforderungen und Rahmenbedingungen anpassen und an vorderster 
Front (Entwicklung von Netzwerken, eHealth) zukunftsorientiert arbeiten.

Und nun wissen «es» $3 / 4$ der Delegierten von Hausärzte Schweiz besser. Ohne Berücksichtigung der bisherigen Fehleinschätzung sämtlicher Führungsorgane, ohne basisdemokratische Legitimierung durch lichen) Vorlage höher gewichtet als das politische Gewicht der Ärzteschaft. Für uns ist dieses ideologische Festhalten an der eigenen Meinung ohne Placet der Basis ein standespolitisches und taktisches Desaster. Es werden Zeiten und Entwicklungen auf uns zukommen, die eine Solidarität aller FMH-Mitglieder

\section{«Uns ist keine Organisation bekannt, bei der eine Mitgliederumfrage eine Zustimmung zur Vorlage ergeben hätte.»}

eine Mitgliederbefragung und ohne Solidarität mit der FMH wird ein Kurs gegen das Referendum beschlossen, wird die Verteidigung einer nur fraglich für die Ärzteschaft nützlichen (und gemäss unserer Einschätzung für die Hausärzte sogar potentiell gefähr- zur Verteidigung effektiver hausärztlicher Interessen erfordern, und es ist unklug zu meinen, alleine wäre man stärker. Das Volk wird das Referendum gutheissen. Die Hausärzte sollten zu den Gewinnern gehören!

Für die Hausärzte-Vertreter im Vorstand Ärztegesellschaft Baselland:

Dr. med. Beat Biedermann, FMH Innere Medizin und Onkologie, Ergolzstrasse 31, 4414 Füllinsdorf

Dr. med. Urs Buess, FMH Allgemeine Medizin, Mühlemattstrasse 39, 4414 Füllinsdorf

Dr. med. Aurelio Corfù, FMH Pädiatrie, Käppelibodenweg 44, 4132 Muttenz

Dr. med. Walter Frommenwiler, FMH Allgemeine Medizin und Chirurgie, Bahnhofstrasse 20, 4402 Frenkendorf

Dr. med. Jürg Jutzi, FMH Innere Medizin, Gewerbestrasse 3, 4416 Bubendorf

Dr. med. Thomas Reichenstein, FMH Innere Medizin, Poststrasse 2, 4302 Augst

Dr. med. Martin Schwab, FMH Allgemeine Medizin, Hauptstrasse 39, 4450 Sissach

Dr. med. Angelika Senst, FMH Allgemeine Medizin, Trottenstrasse 9, 4402 Frenkendorf

Dr. med. Lukas Wagner, FMH Allgemeine Medizin, Hauptstrasse 35, 4127 Birsfelden

Für das Präsidium Ärztegesellschaft Baselland:

Dr. med. Tobias Eichenberger, Präsident, FMH Urologie, Hammerstrasse 35, 4410 Liestal 